\title{
Nonlinear Regression with Censored Data
}

\author{
Cédric Heuchenne $e^{1,2}$ \\ Institut de Statistique \\ Ingrid Van Keilegom ${ }^{1}$ \\ Institut de Statistique \\ Université catholique de Louvain \\ Université catholique de Louvain
}

April 30, 2008

\begin{abstract}
Suppose the random vector $(X, Y)$ satisfies the regression model $Y=m(X)+$ $\sigma(X) \varepsilon$, where $m(\cdot)=E(Y \mid \cdot)$ belongs to some parametric class $\left\{m_{\theta}(\cdot): \theta \in \Theta\right\}$ of regression functions, $\sigma^{2}(\cdot)=\operatorname{Var}(Y \mid \cdot)$ is unknown, and $\varepsilon$ is independent of $X$. The response $Y$ is subject to random right censoring, and the covariate $X$ is completely observed. A new estimation procedure for the true, unknown parameter vector $\theta_{0}$ is proposed, that extends the classical least squares procedure for nonlinear regression to the case where the response is subject to censoring. The consistency and asymptotic normality of the proposed estimator are established. The estimator is compared via simulations with an estimator proposed by Stute in 1999, and both methods are also applied to a fatigue life data set of strain-controlled materials.
\end{abstract}

KEY WORDS: Bandwidth selection; Bootstrap; Fatigue life data; Kernel method; Least squares estimation; Nonparametric regression; Right censoring; Survival analysis.

1 This research was supported by IAP research network grant nr. P5/24 of the Belgian government (Belgian Science Policy).

${ }^{2}$ The first author is now at the University of Liège, HEC-Managment School of ULg. 


\section{Introduction}

Nonlinear models are often used when analysing a possibly censored survival time depending on a covariate. By example, in medical surveys, the relationship between the survival time and the age of a patient who has received a given treatment is often nonlinear and the survival time is subject to right censoring since the patient may decide to leave the study, die due to another cause than the disease from which he suffers or the study itself can be stopped. Therefore, although the methodology proposed in this paper enlarges beyond the following topic, we are here interested in the (nonlinear) relationship between fatigue life of metal, ceramic or composite materials (which is considered as a survival time) and applied stress. This important input to design-for-reliability processes is motivated by the need to develop and present quantitative fatigue-life information used in the design of jet engines. Indeed, according to the air speed that enters an aircraft engine, the fan, the compressor and the turbine rotate at different speeds and therefore are submitted to different stresses. Moreover, fatigue life may be censored since failures may result from impurities or vacuums in the studied materials, or no failure may occur at all due to time constraints of the experiments. In this context, we can thus consider the regression model

$$
Y=m_{\theta_{0}}(X)+\sigma(X) \varepsilon,
$$

where $\sigma^{2}(\cdot)=\operatorname{Var}(Y \mid \cdot), m_{\theta_{0}}(\cdot)=E(Y \mid \cdot)$ is the regression curve, known upto a parameter vector $\theta \in \Theta$ with true unknown value $\theta_{0}, \Theta$ is a compact subset of $\mathbb{R}^{d}$, and the error term $\varepsilon$ is independent of the (one-dimensional) covariate $X$. This model has already been considered in fatigue curve analysis (Nelson 1984, Pascual and Meeker 1997) but with parametric forms for $\sigma^{2}(\cdot)$ and the distribution of $\varepsilon$. Since those forms are not easily determined, it can be important to consider estimation of the conditional mean $m_{\theta_{0}}(\cdot)$ without any parametric constraint on the other quantities of the model (1.1). Suppose also that $Y$ is subject to random right censoring, i.e. instead of observing $Y$, we only observe $(Z, \Delta)$, where $Z=\min (Y, C), \Delta=I(Y \leq C)$ and the random variable $C$ represents the censoring time, which is independent of $Y$, conditionally on $X$. Let $\left(Y_{i}, C_{i}, X_{i}, Z_{i}, \Delta_{i}\right)$ $(i=1, \ldots, n)$ be $n$ independent copies of $(Y, C, X, Z, \Delta)$.

We are interested in the estimation of the parameter vector $\theta_{0}$ by means of an extension to censored data of the classical least squares procedure for nonlinear regression. When the regression curve is polynomial, this estimation problem has been studied extensively in the literature, see e.g. Heuchenne and Van Keilegom (2004) for a literature overview. When the regression curve belongs however to some parametric, but nonlinear family of regression functions, much less research has been devoted to this problem. Stute (1999) proposed the following estimation procedure, which is an extension of his earlier paper 
(Stute (1993)) on linear regression : minimize

$$
\sum_{i=1}^{n} W_{i n}\left\{Z_{(i)}-m_{\theta}\left(X_{(i)}\right)\right\}^{2}
$$

with respect to $\theta$, where

$$
W_{i n}=\frac{\Delta_{(i)}}{n-i+1} \prod_{j=1}^{i-1}\left(\frac{n-j}{n-j+1}\right)^{\Delta_{(j)}}
$$

is the jump size of the bivariate empirical distribution function $\hat{F}(x, y)$ proposed by Stute (1993) :

$$
\hat{F}(x, y)=\sum_{i=1}^{n} W_{i n} I\left(X_{(i)} \leq x, Z_{(i)} \leq y\right),
$$

$Z_{(1)}, \ldots, Z_{(n)}$ are the order statistics of $Z_{1}, \ldots, Z_{n}$, and $X_{(i)}$ and $\Delta_{(i)}$ are the corresponding covariate and censoring indicator (note that for simplicity we have considered untied observations). The method is very easy to implement in practice and, unlike many of the estimation procedures for polynomial regression, it is not based on a, sometimes delicate, tuning or bandwidth parameter. A major drawback of this estimation procedure however, is that it assumes that (1) $Y$ and $C$ are independent (unconditionally on $X$ ) and that (2) $P(Y \leq C \mid X, Y)=P(Y \leq C \mid Y)$, which is satisfied when e.g. $C$ is independent of $X$. Both assumptions are often violated in practice.

In this paper we propose a new estimation method for $\theta_{0}$, which does not require the above two assumptions. The idea of the method is as follows :

1. Estimate the true unknown survival time of a censored observation $\left(X_{i}, Z_{i}, \Delta_{i}=0\right)$ by a nonparametric estimator of $E\left(Y_{i} \mid X_{i}, Y_{i}>Z_{i}\right)$.

2. Estimate $\theta_{0}$ by minimizing the least squares criterium for completely observed data, applied to the 'synthetic' data obtained in step (1).

For polynomial regression models a similar estimation procedure has been studied in Heuchenne and Van Keilegom (2004). The estimation in step (1) is done by using kernel smoothing with an adaptively chosen bandwidth parameter. The details of the proposed method are given in the next section.

The paper is organized as follows. In the next section, the estimation procedure is described in detail. Section 3 summarizes the main asymptotic results, including the asymptotic normality of the estimator. In Section 4 we present the results of a simulation study, in which the new procedure is compared with the method of Stute (1999). Section 5 is devoted to the analysis of data from a study on the relationship between fatigue life of metal and applied stress. The Appendix contains the proofs of the main results of Section 3 . 


\section{Notations and description of the method}

As outlined in the introduction, the idea of the proposed method consists of first estimating the unknown survival times of the censored observations, and second to apply a standard nonlinear least squares procedure on the so-obtained artificial data points. Define

$$
Y_{i}^{*}=Y_{i} \Delta_{i}+E\left[Y_{i} \mid Y_{i}>C_{i}, X_{i}\right]\left(1-\Delta_{i}\right)
$$

and note that $E\left(Y_{i} \mid X_{i}\right)=E\left(Y_{i}^{*} \mid X_{i}\right)=m_{\theta_{0}}\left(X_{i}\right)$. Hence, we can work in the sequel with the variable $Y_{i}^{*}$ instead of with $Y_{i}$. In order to estimate $Y_{i}^{*}$ for a censored observation, we first need to introduce a number of notations.

Let $m^{0}(\cdot)$ be any location function and $\sigma^{0}(\cdot)$ be any scale function, meaning that $m^{0}(x)=T(F(\cdot \mid x))$ and $\sigma^{0}(x)=S(F(\cdot \mid x))$ for some functionals $T$ and $S$ that satisfy $T\left(F_{a Y+b}(\cdot \mid x)\right)=a T\left(F_{Y}(\cdot \mid x)\right)+b$ and $S\left(F_{a Y+b}(\cdot \mid x)\right)=a S\left(F_{Y}(\cdot \mid x)\right)$, for all $a \geq 0$ and $b \in \mathbb{R}$ (here $F_{a Y+b}(\cdot \mid x)$ denotes the conditional distribution of $a Y+b$ given $X=x$ ). Let $\varepsilon^{0}=\left(Y-m^{0}(X)\right) / \sigma^{0}(X)$. Then, it can be easily seen that if model (1.1) holds (i.e. $\varepsilon$ is independent of $X$ ), then $\varepsilon^{0}$ is also independent of $X$.

Define

$F(y \mid x)=P(Y \leq y \mid x)$, the response conditional distribution,

$G(y \mid x)=P(C \leq y \mid x)$, the censoring conditional distribution,

$H(y \mid x)=P(Z \leq y \mid x)(H(y)=P(Z \leq y))$, the observable (un)conditional distribution, $H_{\delta}(y \mid x)=P(Z \leq y, \Delta=\delta \mid x)$, the observable conditional subdistributions for $\delta=0,1$, $F_{\varepsilon}^{0}(y)=P\left(\varepsilon^{0} \leq y\right), S_{\varepsilon}^{0}(y)=1-F_{\varepsilon}^{0}(y)$, the distribution and survival functions of $\varepsilon^{0}$,

and $F_{X}(x)=P(X \leq x)$. For $E^{0}=\left(Z-m^{0}(X)\right) / \sigma^{0}(X)$, we also denote $H_{\varepsilon}^{0}(y)=P\left(E^{0} \leq\right.$ $y), H_{\varepsilon \delta}^{0}(y)=P\left(E^{0} \leq y, \Delta=\delta\right), H_{\varepsilon}^{0}(y \mid x)=P\left(E^{0} \leq y \mid x\right)$ and $H_{\varepsilon \delta}^{0}(y \mid x)=P\left(E^{0} \leq y, \Delta=\right.$ $\delta \mid x)(\delta=0,1)$. The probability density functions of the distributions defined above will be denoted with lower case letters, and $R_{X}$ denotes the support of the variable $X$.

It is easily seen that

$$
Y_{i}^{*}=Y_{i} \Delta_{i}+\left[m^{0}\left(X_{i}\right)+\frac{\sigma^{0}\left(X_{i}\right)}{1-F_{\varepsilon}^{0}\left(E_{i}^{0}\right)} \int_{E_{i}^{0}}^{\infty} y d F_{\varepsilon}^{0}(y)\right]\left(1-\Delta_{i}\right)
$$

for any location function $m^{0}(\cdot)$ and scale function $\sigma^{0}(\cdot)$. The idea is now to choose $m^{0}$ and $\sigma^{0}$ in such a way that they can be estimated consistently. As is well known (see by example Van Keilegom and Veraverbeke (1997)), the right tail of the distribution $F(y \mid \cdot)$ cannot be estimated in a consistent way due to the presence of right censoring. Therefore, 
we work with the following choices of $m^{0}$ and $\sigma^{0}$ :

$$
m^{0}(x)=\int_{0}^{1} F^{-1}(s \mid x) J(s) d s, \quad \sigma^{02}(x)=\int_{0}^{1} F^{-1}(s \mid x)^{2} J(s) d s-m^{02}(x),
$$

where $F^{-1}(s \mid x)=\inf \{y ; F(y \mid x) \geq s\}$ is the quantile function of $Y$ given $x$ and $J(s)$ is a given score function satisfying $\int_{0}^{1} J(s) d s=1$. When $J(s)$ is chosen appropriately (namely put to zero in the right tail, there where the quantile function cannot be estimated in a consistent way due to the right censoring), $m^{0}(x)$ and $\sigma^{0}(x)$ can be estimated consistently. Now, replace the distribution $F(y \mid x)$ in (2.1) by the Beran (1981) estimator, defined by (in the case of no ties) :

$$
\hat{F}(y \mid x)=1-\prod_{Z_{i} \leq y, \Delta_{i}=1}\left\{1-\frac{W_{i}\left(x, a_{n}\right)}{\sum_{j=1}^{n} I\left(Z_{j} \geq Z_{i}\right) W_{j}\left(x, a_{n}\right)}\right\},
$$

where

$$
W_{i}\left(x, a_{n}\right)=\frac{K\left(\frac{x-X_{i}}{a_{n}}\right)}{\sum_{j=1}^{n} K\left(\frac{x-X_{j}}{a_{n}}\right)},
$$

$K$ is a kernel function and $\left\{a_{n}\right\}$ a bandwidth sequence, and define

$$
\hat{m}^{0}(x)=\int_{0}^{1} \hat{F}^{-1}(s \mid x) J(s) d s, \quad \hat{\sigma}^{02}(x)=\int_{0}^{1} \hat{F}^{-1}(s \mid x)^{2} J(s) d s-\hat{m}^{02}(x)
$$

as estimators for $m^{0}(x)$ and $\sigma^{02}(x)$. Next, let

$$
\hat{F}_{\varepsilon}^{0}(y)=1-\prod_{\hat{E}_{(i)}^{0} \leq y, \Delta_{(i)}=1}\left(1-\frac{1}{n-i+1}\right),
$$

denote the Kaplan-Meier (1958)-type estimator of $F_{\varepsilon}^{0}$ (in the case of no ties), where $\hat{E}_{i}^{0}=\left(Z_{i}-\hat{m}^{0}\left(X_{i}\right)\right) / \hat{\sigma}^{0}\left(X_{i}\right), \hat{E}_{(i)}^{0}$ is the $i$-th order statistic of $\hat{E}_{1}^{0}, \ldots, \hat{E}_{n}^{0}$ and $\Delta_{(i)}$ is the corresponding censoring indicator. This estimator has been studied in detail by Van Keilegom and Akritas (1999). This leads to the following estimator of $Y_{i}^{*}$ :

$$
\hat{Y}_{T i}^{*}=Y_{i} \Delta_{i}+\left\{\hat{m}^{0}\left(X_{i}\right)+\frac{\hat{\sigma}^{0}\left(X_{i}\right)}{1-\hat{F}_{\varepsilon}^{0}\left(\hat{E}_{i}^{0 T}\right)} \int_{\hat{E}_{i}^{0 T}}^{\hat{S}_{i}} y d \hat{F}_{\varepsilon}^{0}(y)\right\}\left(1-\Delta_{i}\right),
$$

where $\hat{S}_{i}=\left(T_{X_{i}}-\hat{m}^{0}\left(X_{i}\right)\right) / \hat{\sigma}^{0}\left(X_{i}\right), \hat{E}_{i}^{0 T}=\hat{E}_{i}^{0} \wedge \hat{S}_{i}$, and for any $x, T_{x} \leq T \sigma^{0}(x)+m^{0}(x)$, where $T<\tau_{H_{\varepsilon}^{0}}$ and $\tau_{F}=\inf \{y: F(y)=1\}$ for any distribution $F$. Note that due to the right censoring, we have to truncate the integral in the definition of $\hat{Y}_{T i}^{*}$ (however, when $\tau_{F_{\varepsilon}^{0}} \leq \tau_{G_{\varepsilon}^{0}}$, the bound $\hat{S}_{i}$ can be chosen arbitrarily close to $\tau_{F_{\varepsilon}^{0}}$ for $n$ sufficiently large). Finally, the new data points (2.5) are introduced into the least squares problem

$$
\min _{\theta \in \Theta} \sum_{i=1}^{n}\left[\hat{Y}_{T i}^{*}-m_{\theta}\left(X_{i}\right)\right]^{2} .
$$


In order to focus on the primary issues, we assume the existence of a well-defined minimizer of (2.6). The solution of this problem can be obtained using an (iterative) procedure for nonlinear minimization problems, like e.g. a Newton-Raphson procedure. Denote a minimizer of $(2.6)$ by $\hat{\theta}_{n}^{T}=\left(\hat{\theta}_{n 1}^{T}, \ldots, \hat{\theta}_{n d}^{T}\right)$. As it is clear from the definition of $\hat{Y}_{T i}^{*}, \hat{\theta}_{n 1}^{T}, \ldots, \hat{\theta}_{n d}^{T}$ are actually estimating the unique $\theta_{0}^{T}=\left(\theta_{01}^{T}, \ldots, \theta_{0 d}^{T}\right)$ which minimizes $E\left[\left\{E\left(Y_{T}^{*} \mid X\right)-m_{\theta}(X)\right\}^{2}\right]$ (see hypothesis (A10) in the Appendix), where

$$
Y_{T}^{*}=Y \Delta+\left\{m^{0}(X)+\frac{\sigma^{0}(X)}{1-F_{\varepsilon}^{0}\left(E^{0 T}\right)} \int_{E^{0 T}}^{S_{X}} y d F_{\varepsilon}^{0}(y)\right\}(1-\Delta),
$$

$S_{X}=\left(T_{X}-m^{0}(X)\right) / \sigma^{0}(X)$ and $E^{0 T}=\left(Z \wedge T_{X}-m^{0}(X)\right) / \sigma^{0}(X)=E^{0} \wedge S_{X}$. As before, these coefficients $\theta_{01}^{T}, \ldots, \theta_{0 d}^{T}$ can be made arbitrarily close to $\theta_{01}, \ldots, \theta_{0 d}$, provided $\tau_{F_{\varepsilon}^{0}} \leq \tau_{G_{\varepsilon}^{0}}$.

\section{Asymptotic results}

We start by showing the convergence in probability of $\hat{\theta}_{n}^{T}$ and of the least squares criterion function. This will allow us to develop an asymptotic representation for $\hat{\theta}_{n j}^{T}-\theta_{0 j}^{T}(j=$ $1, \ldots, d)$, which in turn will give rise to the asymptotic normality of these estimators. The assumptions and notations used in the results below, as well as the proof of the two first results, are given in the Appendix.

Theorem 3.1 Assume (A1) (i)-(iii), (A2) (i), (ii), (A3) (i), (ii), (A4) (i), (A6), (A10) and $m_{\theta}(x)$ is continuous in $(x, \theta)$. Let $S_{n}(\theta)=\frac{1}{n} \sum_{i=1}^{n}\left[\hat{Y}_{T i}^{*}-m_{\theta}\left(X_{i}\right)\right]^{2}$. Then,

$$
\hat{\theta}_{n}^{T}-\theta_{0}^{T}=o_{P}(1)
$$

and

$$
S_{n}\left(\hat{\theta}_{n}^{T}\right)=E\left[\sigma^{02}(X) \operatorname{Var}\left(\varepsilon_{T}^{0 *} \mid X\right)\right]+E\left[\left\{E\left(Y_{T}^{*} \mid X\right)-m_{\theta_{0}^{T}}(X)\right\}^{2}\right]+o_{P}(1),
$$

where

$$
\varepsilon_{T}^{0 *}=\varepsilon^{0} \Delta+\frac{1}{1-F_{\varepsilon}^{0}\left(E^{0 T}\right)} \int_{E^{0 T}}^{S_{X}} u d F_{\varepsilon}^{0}(u)(1-\Delta) .
$$

Theorem 3.2 Assume (A1)-(A10). Then,

$$
\hat{\theta}_{n}^{T}-\theta_{0}^{T}=\Omega^{-1} n^{-1} \sum_{i=1}^{n} \rho\left(X_{i}, Z_{i}, \Delta_{i}\right)+\left(\begin{array}{c}
o_{P}\left(n^{-1 / 2}\right) \\
\vdots \\
o_{P}\left(n^{-1 / 2}\right)
\end{array}\right),
$$


where $\Omega=\left(\Omega_{j k}\right)(j, k=1, \ldots, d)$,

$$
\Omega_{j k}=E\left[\frac{\partial m_{\theta_{0}^{T}}(X)}{\partial \theta_{j}} \frac{\partial m_{\theta_{0}^{T}}(X)}{\partial \theta_{k}}-\left\{Y_{T}^{*}-m_{\theta_{0}^{T}}(X)\right\} \frac{\partial^{2} m_{\theta_{0}^{T}}(X)}{\partial \theta_{j} \partial \theta_{k}}\right],
$$

$\rho=\left(\rho_{1}, \ldots, \rho_{d}\right)^{\prime}$

$$
\begin{aligned}
\rho_{j}\left(X_{i}, Z_{i}, \Delta_{i}\right)= & \int_{R_{X}} \frac{\partial m_{\theta_{0}^{T}}(x)}{\partial \theta_{j}} \sigma^{0}(x) \int\left\{\frac{\varphi\left\{X_{i}, Z_{i}, \Delta_{i}, e_{x}^{0 T}(z)\right\}}{\left[1-F_{\varepsilon}^{0}\left\{e_{x}^{0 T}(z)\right\}\right]^{2}} \int_{e_{x}^{0 T}(z)}^{S_{x}} u d F_{\varepsilon}^{0}(u)\right. \\
& \left.+\frac{1}{1-F_{\varepsilon}^{0}\left\{e_{x}^{0 T}(z)\right\}} \int_{e_{x}^{0 T}(z)}^{S_{x}} u d \varphi\left(X_{i}, Z_{i}, \Delta_{i}, u\right)\right\} d H_{0}(z \mid x) d F_{X}(x) \\
& +f_{X}\left(X_{i}\right) \int B_{j}\left(z, Z_{i}, \Delta_{i} \mid X_{i}\right) d H_{0}\left(z \mid X_{i}\right)+\frac{\partial m_{\theta_{0}^{T}}\left(X_{i}\right)}{\partial \theta_{j}}\left(Y_{T i}^{*}-m_{\theta_{0}^{T}}\left(X_{i}\right)\right)
\end{aligned}
$$

$(j=1, \ldots, d ; i=1, \ldots, n)$.

Theorem 3.3 Under the assumptions of Theorem 3.2, $n^{1 / 2}\left(\hat{\theta}_{n}^{T}-\theta_{0}^{T}\right) \stackrel{d}{\rightarrow} N(0, \Sigma)$, where

$$
\Sigma=\Omega^{-1} E\left[\rho(X, Z, \Delta) \rho^{\prime}(X, Z, \Delta)\right] \Omega^{-1}
$$

The proof of this result follows readily from Theorem 3.2.

Remark 3.4 Note that when model (1.1) is polynomial, the representation in Theorem 3.2 reduces to the one obtained by Heuchenne and Van Keilegom (2004). Note that when the model is polynomial, $\boldsymbol{\beta}_{T}=\left(\beta_{T 0}, \ldots, \beta_{T p}\right)=\left(\mathcal{X}^{\prime} \mathcal{X}\right)^{-1} \mathcal{X}^{\prime} E\left(Y_{T i}^{*} \mid X_{i}\right)_{i=1}^{n}$ (conditionally on $\left.X_{1}, \ldots, X_{n}\right)$.

\section{Practical implementation and simulations}

\subsection{Practical implementation}

The estimator $\hat{\theta}_{n}^{T}$ depends on a number of parameters, namely on the bandwidth $a_{n}$, the score function $J$, and the cut off point $T$. All of them can be chosen in a data driven way. First, for the score function $J$, we recommend the choice $J(s)=b^{-1} I(0 \leq s \leq$ b) $(0 \leq s \leq 1)$, where $b=\min _{1 \leq i \leq n} \hat{F}\left(+\infty \mid X_{i}\right)$. In this way, the region where the Beran estimators $\hat{F}\left(\cdot \mid X_{1}\right), \ldots, \hat{F}\left(\cdot \mid X_{n}\right)$ are inconsistent is not used, and on the other hand, we exploit to a maximum the 'consistent' region.

A number of adaptive procedures can be used to select the bandwidth $a_{n}$, depending on the criterion function one has in mind. If the goal would be to optimize the estimation 
of $m^{0}$ and $\sigma^{0}$, one could use e.g. a bootstrap approach that selects the bandwidth that minimizes the estimated MSE of their estimators. On the other hand, it seems more appropriate here to choose the bandwidth in function of the end goal, namely in order to optimize the estimation of $\theta$. We therefore prefer to choose the bandwidth by minimizing the least squares criterium function

$$
\sum_{i=1}^{n}\left\{\hat{Y}_{T i}^{*}\left(a_{n}\right)-m_{\hat{\theta}_{n}^{T}\left(a_{n}\right)}\left(X_{i}\right)\right\}^{2}
$$

over a grid of $a_{n}$-values. Note that we have added the argument $a_{n}$ to $\hat{Y}_{T i}^{*}$ and $\hat{\theta}_{n}^{T}(i=$ $1, \ldots, n)$ in order to emphasize the dependence on $a_{n}$ of these quantities. We illustrate this procedure to select the bandwidth in the next subsection.

Finally, $\hat{S}_{i}(i=1, \ldots, n)$ can be chosen larger (or equal) than the last order statistic $\hat{E}_{(n)}^{0}$ of the estimated residuals $\hat{E}_{i}^{0}, i=1, \ldots, n$. In this way, all the Kaplan-Meier jumps of the integral (2.5) are considered.

As an alternative to the normal approximation obtained in the previous section, a bootstrap procedure can be used to approximate the distribution of $\hat{\theta}_{n}^{T}$. The procedure proposed by Li and Datta (2001) can be used for this. It extends Efron's (1981) procedure developed for censored data to the nonparametric regression context. If in addition one wants to take advantage of the validity of model (1.1), a more elaborate procedure can be used, in which the survival times are drawn under model (1.1) (instead of from a nonparametric estimator of their conditional distribution).

\subsection{Simulations}

We compare now the finite sample behavior of Stute's (1999) estimator with the newly proposed estimator. We are primarily interested in the behavior of the bias and variance of the two estimators. The simulations are carried out for samples of size $n=100$ and the results are obtained by using 250 simulations.

In the first setting, we generate i.i.d. data from the normal homoscedastic regression model

$$
Y=\frac{\theta_{0}}{11} \sin \left(\theta_{1} X^{2}\right)+\sigma \varepsilon
$$

where $\theta_{0}=\theta_{1}=11, \sigma^{2}=0.5$ or $1, X$ has a uniform distribution on the unit interval and the error term $\varepsilon$ is a standard normal random variable. The censoring variable $C$ satisfies $C=\left(\alpha_{0} / 11\right) \sin \left(\alpha_{1} X^{2}\right)+\sigma \varepsilon^{*}$, for certain choices of $\alpha_{0}$ and $\alpha_{1}$ and where $\varepsilon^{*}$ has a standard normal distribution. We further assume that $\varepsilon$ and $\varepsilon^{*}$ are independent of $X$, and that $\varepsilon$ is independent of $\varepsilon^{*}$. It is easy to see that, under this model,

$$
P(\Delta=0 \mid X=x)=1-\Phi\left(\frac{\left(\alpha_{0} / 11\right) \sin \left(\alpha_{1} x^{2}\right)-\left(\theta_{0} / 11\right) \sin \left(\theta_{1} x^{2}\right)}{\sqrt{2} \sigma}\right) .
$$


We compare here the new method for homoscedastic errors, with Stute's method. Note that the conditions of the latter method, which are outlined in Section 1 (i.e. $Y$ and $C$ are independent and $P(Y \leq C \mid X, Y)=P(Y \leq C \mid Y))$ are not satisfied for this model.

We work with a biquadratic kernel function $K(x)=(15 / 16)\left(1-x^{2}\right)^{2} I(|x| \leq 1)$. In order to improve the behavior near the boundaries of the covariate space, we work with the boundary corrected kernels proposed by Müller and Wang (1994). Since these kernels can become negative, it may happen that the Beran estimator decreases at certain time points. In these cases, the estimator is redefined as being constant until it starts increasing again.

For the two methods, the Levenberg-Marquardt algorithm (Levenberg (1944) and Marquardt (1963)) is used to solve equations (1.2) and (2.6) (for a fixed value of the bandwidth parameter).

The bandwidth $a_{n}$ is selected by minimizing expression (4.1) over a grid of 20 possible bandwidths between 0 and 1 . For small values of $a_{n}$, the window $\left[x-a_{n}, x+a_{n}\right]$ at a point $x$ might not contain any $X_{i}(i=1, \ldots, n)$ for which the corresponding $Y_{i}$ is uncensored (and in that case estimation of $F(\cdot \mid x)$ is impossible). We enlarge the window in that case such that it contains at least one uncensored data point in its interior. It might also happen that the bandwidth $a_{n}$ at a point $x$ is larger than the distance from $x$ to both the left and right endpoint of the interval. In such cases, the bandwidth is redefined as the maximum of these two distances.

Table 1 summarizes the simulation results for different values of $\alpha_{0}, \alpha_{1}$ and $\sigma$. For fixed value of $\sigma$, the values of $\alpha_{0}$ and $\alpha_{1}$ are chosen in such a way that some variation in the censoring probability curves is obtained (different proportions of censoring, different degrees of smoothness of the censoring probability curve,...). The proportion of censoring (in $\%$ and denoted $\mathrm{CP}$ in the tables) is computed as the average of $P(\Delta=0 \mid x)$ for an equispaced grid of values of $x$.

In the second setting, we generate i.i.d. data from the normal homoscedastic regression model

$$
Y=\frac{5}{4} \exp \left(\theta_{0} X+\theta_{1} X^{2}\right)+\sigma \varepsilon
$$

where $\theta_{0}=0.8$ and $\theta_{1}=1$. The other quantities in model (4.3) are chosen as in (4.2). The censoring variable $C$ satisfies $C=\alpha_{0} \exp \left(\alpha_{1} X+\alpha_{2} X^{2}\right)+\sigma \varepsilon^{*}$, with the same characteristics as in the first setting. Table 2 summarizes the simulation results for different values of $\alpha_{0}, \alpha_{1}, \alpha_{2}$ and $\sigma$ chosen as for Table 1 . Again we compare the new method for homoscedastic errors with Stute's estimator, whose assumptions on the survival and censoring variables are not satisfied here. 


\begin{tabular}{|cc|ccccccc|}
\hline$\alpha_{0}$ & $\alpha_{1}$ & \multicolumn{4}{|c}{$\hat{\theta}_{0}$} & & \multicolumn{3}{c|}{$\hat{\theta}_{1}$} \\
\cline { 8 - 9 }$\sigma^{2}$ & CP & Bias & Var & MSE & & Bias & Var & MSE \\
\hline 24 & 1.6 & -0.56 & 4.340 & 4.652 & & -0.06 & 0.209 & 0.212 \\
1 & 34.37 & -0.36 & 2.808 & 2.940 & & 0.034 & 0.116 & 0.117 \\
\hline 11 & 11 & 1.271 & 3.669 & 5.285 & & 0.160 & 0.240 & 0.266 \\
1 & 50 & -0.58 & 3.046 & 3.377 & & 0.043 & 0.169 & 0.171 \\
\hline 11 & 12 & 1.258 & 3.909 & 5.493 & & 0.350 & 0.284 & 0.406 \\
1 & 51.12 & -0.61 & 3.175 & 3.544 & & 0.023 & 0.198 & 0.199 \\
\hline 24 & 1.6 & -0.49 & 2.043 & 2.285 & & -0.04 & 0.081 & 0.083 \\
0.5 & 32.73 & -0.16 & 1.660 & 1.686 & & 0.021 & 0.052 & 0.052 \\
\hline 11 & 11 & 0.701 & 1.799 & 2.290 & & 0.136 & 0.120 & 0.138 \\
0.5 & 50 & -0.44 & 1.641 & 1.836 & & 0.027 & 0.088 & 0.089 \\
\hline 11 & 12 & 0.637 & 2.078 & 2.484 & & 0.300 & 0.152 & 0.242 \\
0.5 & 51.52 & -0.46 & 1.581 & 1.794 & & -0.01 & 0.113 & 0.113 \\
\hline
\end{tabular}

Table 1: Results for the Stute estimator (first line) and the new estimator (second line) for model (4.2).

\begin{tabular}{|ccc|cccccccc|}
\hline$\alpha_{0}$ & $\alpha_{1}$ & $\alpha_{2}$ & \multicolumn{4}{|c}{$\hat{\theta}_{0}$} & & \multicolumn{3}{c|}{$\hat{\theta}_{1}$} \\
$\sigma^{2}$ & CP & & Bias & Var & MSE & & Bias & Var & MSE \\
\hline 1.25 & 1.1 & 1 & -0.52 & 0.081 & 0.349 & & 0.549 & 0.104 & 0.406 \\
1 & 33.98 & & 0.002 & 0.077 & 0.077 & & -0.01 & 0.098 & 0.098 \\
\hline 1.25 & 0.8 & 1 & -0.71 & 0.142 & 0.646 & & 0.711 & 0.173 & 0.678 \\
1 & 50 & & 0.026 & 0.088 & 0.088 & & -0.05 & 0.111 & 0.113 \\
\hline 1.25 & 0.2 & 1.65 & -0.91 & 0.173 & 0.995 & & 0.912 & 0.208 & 1.040 \\
1 & 55.22 & & 0.033 & 0.108 & 0.109 & & -0.05 & 0.132 & 0.135 \\
\hline 1.25 & 1.05 & 1 & -0.36 & 0.038 & 0.164 & & 0.376 & 0.049 & 0.190 \\
0.5 & 32.27 & & 0.011 & 0.039 & 0.039 & & -0.02 & 0.049 & 0.050 \\
\hline 1.25 & 0.8 & 1 & -0.53 & 0.064 & 0.345 & & 0.524 & 0.081 & 0.355 \\
0.5 & 50 & & 0.030 & 0.045 & 0.046 & & -0.04 & 0.057 & 0.059 \\
\hline 1.25 & 0.25 & 1.65 & -0.66 & 0.077 & 0.516 & & 0.672 & 0.094 & 0.546 \\
0.5 & 53.62 & & 0.046 & 0.056 & 0.058 & & -0.06 & 0.069 & 0.072 \\
\hline
\end{tabular}

Table 2: Results for the Stute estimator (first line) and the new estimator (second line) for model (4.3). 
In the third setting we consider a normal heteroscedastic regression model

$$
Y=\theta_{0} X+\sin \left(\theta_{1} X\right)+\gamma X \varepsilon
$$

with $\theta_{0}=1, \theta_{1}=10, X$ has a uniform distribution on $[0,1], \varepsilon$ has a standard normal distribution, and $\gamma$ equals $1,2,3$ or 4 . The censoring variable is given by $C=\alpha_{0} X+$ $\sin \left(\alpha_{1} X\right)+\gamma \varepsilon^{*}$, where $\varepsilon^{*}$ has a standard normal distribution. We further assume that $\varepsilon$ and $\varepsilon^{*}$ are independent of $X$, and that $\varepsilon$ is independent of $\varepsilon^{*}$. The assumptions of Stute's method outlined in the introduction are again not satisfied here. The assumptions of the new method are satisfied. Since the model is heteroscedastic, we estimate here the scale function $\sigma(\cdot)$. Table 3 summarizes the simulation results for increasing values of $\gamma$, approximately constant proportion of censoring and approximately the same shape of censoring probability curve.

\begin{tabular}{|cc|ccccccc|}
\hline \multirow{2}{*}{$\alpha_{0}$} & $\alpha_{1}$ & \multicolumn{4}{|c}{$\hat{\theta}_{0}$} & & \multicolumn{3}{c|}{$\hat{\theta}_{1}$} \\
\cline { 3 - 4 } \cline { 8 - 9 }$\gamma$ & $\mathrm{CP}$ & Bias & Var & MSE & & Bias & Var & MSE \\
\hline 1.2 & 11 & -0.12 & 0.044 & 0.059 & & 0.279 & 0.090 & 0.168 \\
1 & 49.54 & -0.05 & 0.037 & 0.039 & & 0.038 & 0.066 & 0.068 \\
\hline 1.2 & 12 & -0.17 & 0.171 & 0.200 & & 0.278 & 1.091 & 1.168 \\
2 & 50.84 & -0.17 & 0.124 & 0.154 & & 0.022 & 0.694 & 0.694 \\
\hline 1.2 & 13.7 & -0.15 & 0.416 & 0.438 & & -0.72 & 4.166 & 4.678 \\
3 & 50.33 & -0.27 & 0.286 & 0.358 & & -0.16 & 1.560 & 1.589 \\
\hline 1.2 & 14.3 & -0.16 & 0.669 & 0.695 & & -1.14 & 5.227 & 6.518 \\
4 & 49.99 & -0.31 & 0.478 & 0.572 & & -0.42 & 3.025 & 3.205 \\
\hline
\end{tabular}

Table 3: Results for the Stute estimator (first line) and the new estimator (second line) for model (4.4).

Tables 1 till 3 show that the new method outperforms Stute's estimator when the restrictive conditions of Stute's procedure are not satisfied. This is especially reflected in the bias, which is in most cases quite large in comparison with the new method. Figure 1 illustrates similar trends for pointwise estimations : when the proportion of censoring increases, the new method keeps stable curves whereas curves of Stute deteriorate more and more, especially for the distance with the true curve. For each method, the mean line (the average at each value of the covariate of the 250 estimated conditional means) lies between two curves which contain at each value of the covariate (a grid of 100 points) $90 \%$ of the estimated conditional means (by taking for each value of the grid the $5 \%$ and $95 \%$ quantiles of the empirical distribution constructed with the estimated conditional 


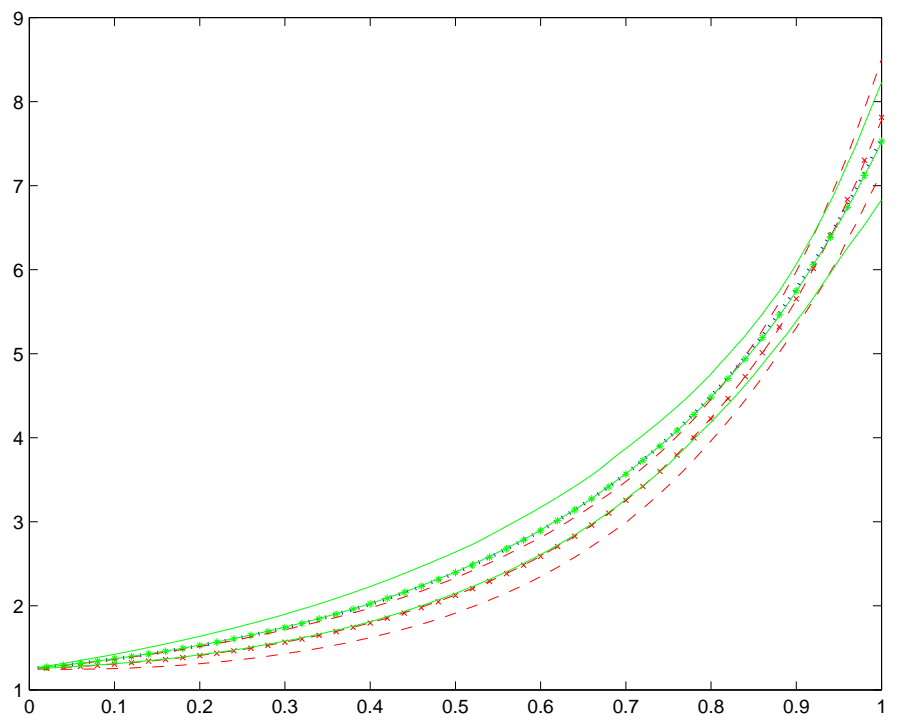

(a)

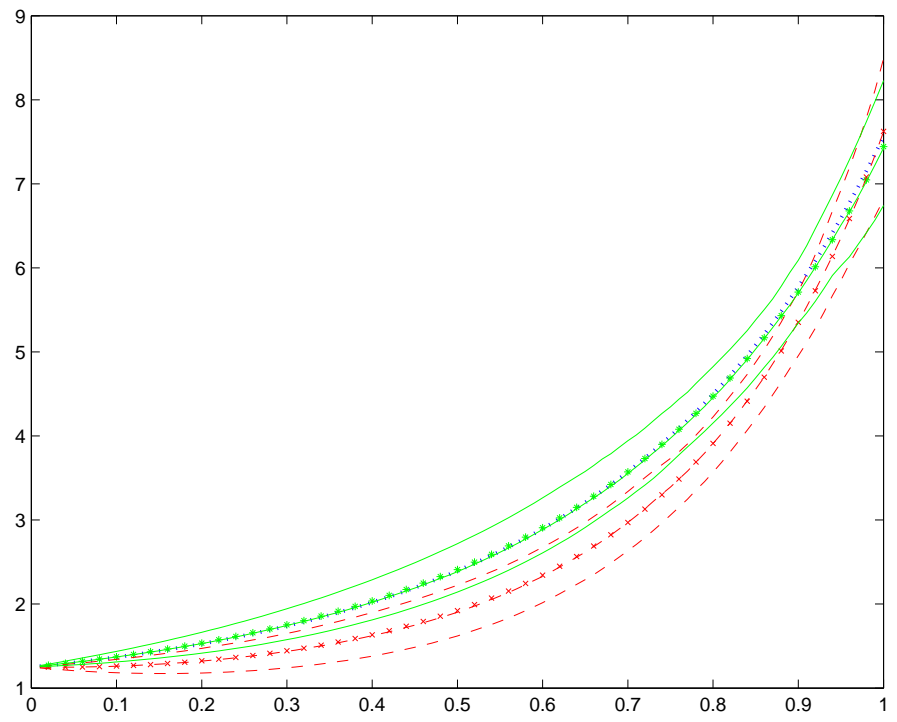

(b)

Figure 1: Comparison of pointwise biases and variabilities of Stute's method with the new one. The solid, respectively, dashed curves are obtained from the new, respectively, Stute's method. Dots indicate the true curve and $*$, respectively, $\times$ represent the mean curve for the new, respectively, Stute's method. (a) First simulation of Table 2; (b) Third simulation of Table 2 . 


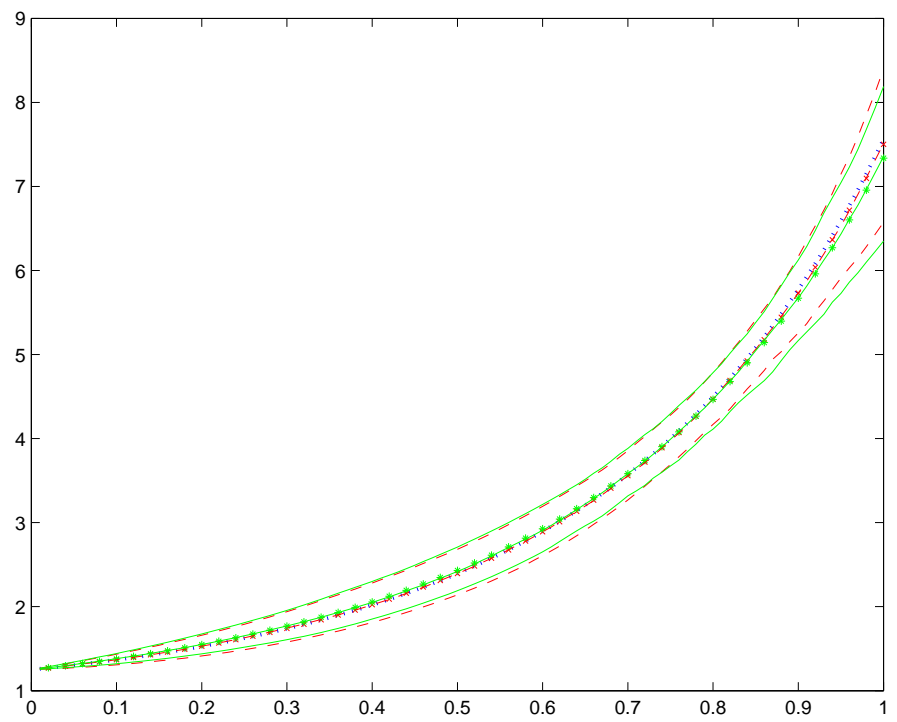

(a)

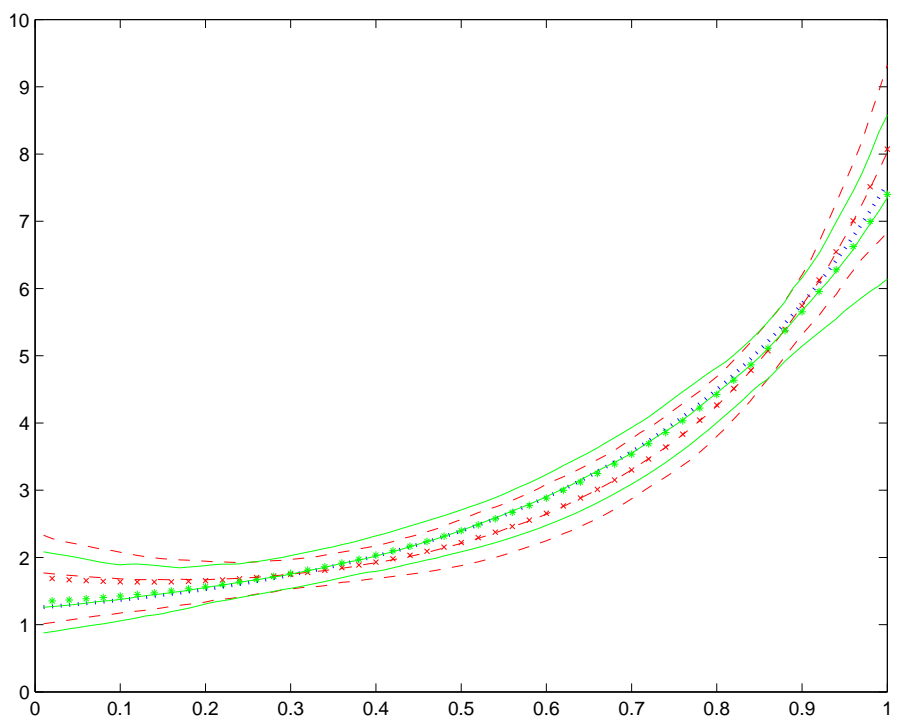

(b)

Figure 2: Comparison of pointwise biases and variabilities of Buckley-James method with the new one. The solid, respectively, dashed curves are obtained from the new, respectively, Stute's method. Dots indicate the true curve and $*$, respectively, $\times$ represent the mean curve for the new, respectively, Stute's method. (a) First simulation of Table 4; (b) First simulation of Table 5. 
means). In the same way, the median curve is also added to show symmetric behaviour of the distributions of the conditional means.

Let us now consider a homoscedastic model, in which $C$ and $X$ are independent :

$$
Y=\theta_{0} \exp \left(\theta_{1} X+\theta_{2} X^{2}\right)+\sigma \varepsilon
$$

where $\theta_{0}=1.25, \theta_{1}=0.8, \theta_{2}=1$, and $X, \varepsilon$ and $\sigma$ are as in model (4.2). The censoring variable $C$ satisfies $C=\alpha_{0}+\rho \varepsilon^{*}$ for some $\alpha_{0}$ and $\rho$, where $\varepsilon^{*}$ is the same as before. This model equals model (4.3) except that the censoring variable is independent of $X$ here. It is easily seen that Stute's assumptions are satisfied in that case. The results in Tables 4 and 5 show more moderated results. For two parameters to estimate, Stute's method behaves slightly better than the new one while when the new method can fit three parameters, its tuning ability (thanks to the bandwidth parameter in the preliminary smoothing) allows to outperform Stute's method. This is also reflected in Figure 2.

\begin{tabular}{|cc|cccccccc|}
\hline \multirow{2}{*}{$\alpha_{0}$} & $\rho$ & \multicolumn{4}{|c}{$\hat{\theta}_{1}$} & & & \multicolumn{3}{c|}{$\hat{\theta}_{2}$} \\
\cline { 3 - 4 } \cline { 8 - 10 }$\sigma^{2}$ & $\mathrm{CP}$ & Bias & Var & MSE & & Bias & Var & MSE \\
\hline 7.5 & 10 & 0.003 & 0.098 & 0.098 & & -0.01 & 0.131 & 0.131 \\
1 & 33.04 & 0.086 & 0.095 & 0.103 & & -0.12 & 0.130 & 0.144 \\
\hline 2.7 & 15 & -0.01 & 0.139 & 0.139 & & -0.00 & 0.186 & 0.186 \\
1 & 50.87 & 0.162 & 0.137 & 0.164 & & -0.22 & 0.192 & 0.240 \\
\hline 7 & 17 & 0.009 & 0.055 & 0.055 & & -0.02 & 0.073 & 0.073 \\
0.5 & 40.82 & 0.087 & 0.056 & 0.063 & & -0.12 & 0.077 & 0.091 \\
\hline 0 & 13 & 0.012 & 0.084 & 0.085 & & -0.02 & 0.118 & 0.119 \\
0.5 & 59.12 & 0.173 & 0.081 & 0.111 & & -0.24 & 0.119 & 0.175 \\
\hline
\end{tabular}

Table 4: Results for the Stute estimator (first line) and the new estimator (second line) for model (4.5).

Table 6 shows the relative performance of the new estimator and the one of Stute for small samples. Let $n=30$ and consider the model

$$
Y=\theta_{0} \exp \left(\theta_{1} X+\theta_{2} X^{2}\right)+(\gamma X+0.1) \varepsilon
$$

where $\theta_{0}=1.25, \theta_{1}=0.8 \theta_{2}=1$, and $\gamma=1,2,3$ or 4 . The other quantities of (4.6) are chosen as in (4.2) and the censoring variable $C$ satisfies $C=\alpha_{0} \exp \left(\alpha_{1} X+\alpha_{2} X^{2}\right)+\gamma \varepsilon^{*}$ for some $\alpha_{0}, \alpha_{1}, \alpha_{2}$ and $\varepsilon^{*}$ chosen as in the other models. Table 6 shows that also for small samples the new method performs well. This can also be explained by an increasing of tuning ability for the new method when reducing the sample size. 


\begin{tabular}{|c|c|c|c|c|c|c|c|c|c|c|}
\hline \multirow{2}{*}{$\begin{array}{l}\alpha_{0} \\
\sigma^{2}\end{array}$} & \multirow{2}{*}{$\begin{array}{c}\rho \\
\mathrm{CP}\end{array}$} & \multicolumn{3}{|c|}{$\hat{\theta}_{0}$} & \multicolumn{3}{|c|}{$\hat{\theta}_{1}$} & \multicolumn{3}{|c|}{$\hat{\theta}_{2}$} \\
\hline & & Bias & Var & MSE & Bias & Var & MSE & Bias & Var & MSE \\
\hline 7.5 & 10 & 0.457 & 0.201 & 0.410 & -1.20 & 1.328 & 2.768 & 0.987 & 0.904 & 1.879 \\
\hline 1 & 33.04 & 0.090 & 0.140 & 0.148 & -0.08 & 1.082 & 1.089 & 0.022 & 0.742 & 0.743 \\
\hline 2.7 & 15 & 0.452 & 0.189 & 0.393 & -1.23 & 1.213 & 2.716 & 1.022 & 0.862 & 1.906 \\
\hline 1 & 50.87 & 0.045 & 0.157 & 0.159 & 0.160 & 1.291 & 1.317 & -0.22 & 0.927 & 0.975 \\
\hline 7 & 17 & 0.493 & 0.160 & 0.403 & -1.34 & 1.117 & 2.921 & 1.114 & 0.789 & 2.031 \\
\hline 0.5 & 40.82 & 0.071 & 0.100 & 0.105 & -0.03 & 0.794 & 0.795 & -0.03 & 0.561 & 0.562 \\
\hline 0 & 13 & 0.359 & 0.136 & 0.265 & -0.28 & 1.218 & 1.297 & -0.14 & 0.934 & 0.952 \\
\hline 0.5 & 59.12 & -0.01 & 0.084 & 0.084 & 0.315 & 0.823 & 0.922 & -0.37 & 0.613 & 0.748 \\
\hline
\end{tabular}

Table 5: Results for the Stute estimator (first line) and the new estimator (second line) for model (4.5).

\begin{tabular}{|c|c|c|c|c|c|c|c|c|c|c|c|}
\hline \multirow{2}{*}{$\begin{array}{l}\alpha_{0} \\
\sigma^{2}\end{array}$} & \multirow{2}{*}{$\begin{array}{c}\alpha_{1} \\
\gamma\end{array}$} & \multirow{2}{*}{$\begin{array}{l}\alpha_{2} \\
\mathrm{CP}\end{array}$} & \multicolumn{3}{|c|}{$\hat{\theta}_{0}$} & \multicolumn{3}{|c|}{$\hat{\theta}_{1}$} & \multicolumn{3}{|c|}{$\hat{\theta}_{2}$} \\
\hline & & & Bias & Var & MSE & Bias & Var & MSE & Bias & Var & MSE \\
\hline 1.25 & 1.1 & 1 & 0.544 & 0.231 & 0.526 & -1.51 & 1.233 & 3.513 & 1.244 & 0.923 & 2.471 \\
\hline 1 & 1 & 32.77 & 0.232 & 0.148 & 0.202 & -0.49 & 1.307 & 1.544 & 0.359 & 1.043 & 1.172 \\
\hline 1.25 & 1.4 & 0.9 & 0.516 & 0.351 & 0.617 & -1.62 & 2.560 & 5.175 & 1.400 & 2.137 & 4.096 \\
\hline 1 & 2 & 33 & 0.242 & 0.270 & 0.328 & -0.47 & 2.665 & 2.882 & 0.340 & 2.227 & 2.343 \\
\hline 1.25 & 1.6 & 0.9 & 0.457 & 0.690 & 0.899 & -1.49 & 6.397 & 8.628 & 1.384 & 5.343 & 7.259 \\
\hline 1 & 3 & 33.06 & 0.266 & 0.450 & 0.521 & -0.39 & 5.736 & 5.887 & 0.268 & 4.860 & 4.932 \\
\hline 1.25 & 1.8 & 0.9 & 0.427 & 1.013 & 1.196 & -1.47 & 8.124 & 10.28 & 1.489 & 7.377 & 9.593 \\
\hline 1 & 4 & 32.65 & 0.276 & 0.749 & 0.825 & -0.18 & 11.20 & 11.23 & 0.118 & 9.176 & 9.190 \\
\hline
\end{tabular}

Table 6: Results for the Stute estimator (first line) and the new estimator (second line) for model (4.6) and $n=30$. 
Finally, other simulations (not reported here) show that models different from the ones considered here, lead to similar simulation results : whenever the restrictive conditions of Stute's method are satisfied, his method can in some cases outperform the new one, whereas the new method behaves considerably better than Stute's estimator in situations where these conditions are not satisfied. Two main arguments explain these results and enable to turn towards a choice of a method to use. First, simulations are achieved for different levels of dependency between $X$ and $C$ according to the importance of the error term in the censoring variable and, second, the dependence of the new data points on a bandwidth $a_{n}$ can be considered as a considerable advantage for the new method since it allows to fine-tune the new estimation procedure. In the univariate case, Stute's method behaves slightly better than the new one only in the case of complete independence between $X$ and $C$. Moreover, even in this case, the tuning ability of the new method (depending on the number of parameters to estimate, the proportion of censoring, the number of data points...) sometimes provides better results than Stute's method. Therefore, it seems that the new method can be recommended in a large range of cases.

\section{Data analysis}

We present, in this section, a set of low-cycle fatigue life data for a strain-controlled test on 26 cylindrical specimens of a nickel-base superalloy. The data were originally described and analyzed in Nelson (1984), and can also be found in Meeker and Escobar (1998). Figure 3 shows the $\log$ of the number of cycles before failure against the pseudostress (Young's modulus times strain). Four censored data are observed; a data point is censored if failure occurs in the radius, weld or threads (censoring coming from impurities or vacuums) or if no failure occurs at all. Therefore, it may be reasonable to think that censoring depends on pseudostress. So, the assumptions of Stute's (1999) procedure (i.e. $Y$ independent of $C$ and $P(Y \leq C \mid X, Y)=P(Y \leq C \mid Y))$ are possibly not satisfied. Moreover, the data seem to follow a heteroscedastic model.

A model often used in the literature (see Pascual and Meeker (1997)) is given by

$$
\log Y=\beta_{0}+\beta_{1} \log (X-\gamma)+\sigma(X) \varepsilon \quad(X>\gamma)
$$

where $\varepsilon$ is independent of $X$ and $E[\varepsilon]=0$. Unlike Pascual and Meeker (1997), we do not impose any parametric form for $\sigma(\cdot)$ and the distribution of $\varepsilon$. This has obviously the advantage of being more robust and flexible (i.e. in some way of validating or rejecting Pascual and Meeker's method), although Pascual and Meeker's completely parametric model will be more efficient in some particular situations (i.e. when their parametric assumptions for the error term are true). Both methods, however, are easily implemented 
and enable to predict $Y$-values for a given $X$-value.

Note that our method does not require the estimation of the variance function $\sigma^{2}(\cdot)$ (since we work with $\sigma^{02}(\cdot)$ in the estimation procedure). However, $\sigma^{2}(\cdot)$ is easily estimated by using the following idea, in analogy with the idea developed in Heuchenne and Van Keilegom (2005b) and Heuchenne (2005) for the nonparametric estimation of $E[\varphi(Y, X) \mid X=x]$, where $\varphi(\cdot, \cdot)$ is some fixed function. It suffices to construct new functions $\varphi^{*}$ in a similar way as in $(2.5)$, where $\varphi(Y, X)=(Y-m(X))^{2}$ and $m(\cdot)=E[Y \mid \cdot]$ is estimated with the method proposed by Heuchenne and Van Keilegom (2005b). The nonparametric estimation of $\sigma^{2}(\cdot)$ can then be achieved by constructing a Nadaraya-Watson estimator based on the estimated realizations of $\varphi^{*}$. This estimator won't be used to compute the variance of a prediction given $X=x$, but to find a close parametric form. In this respect, this would enable to validate or correct the parametric form of $\sigma^{2}(\cdot)$ used in Pascual and Meeker (1997).

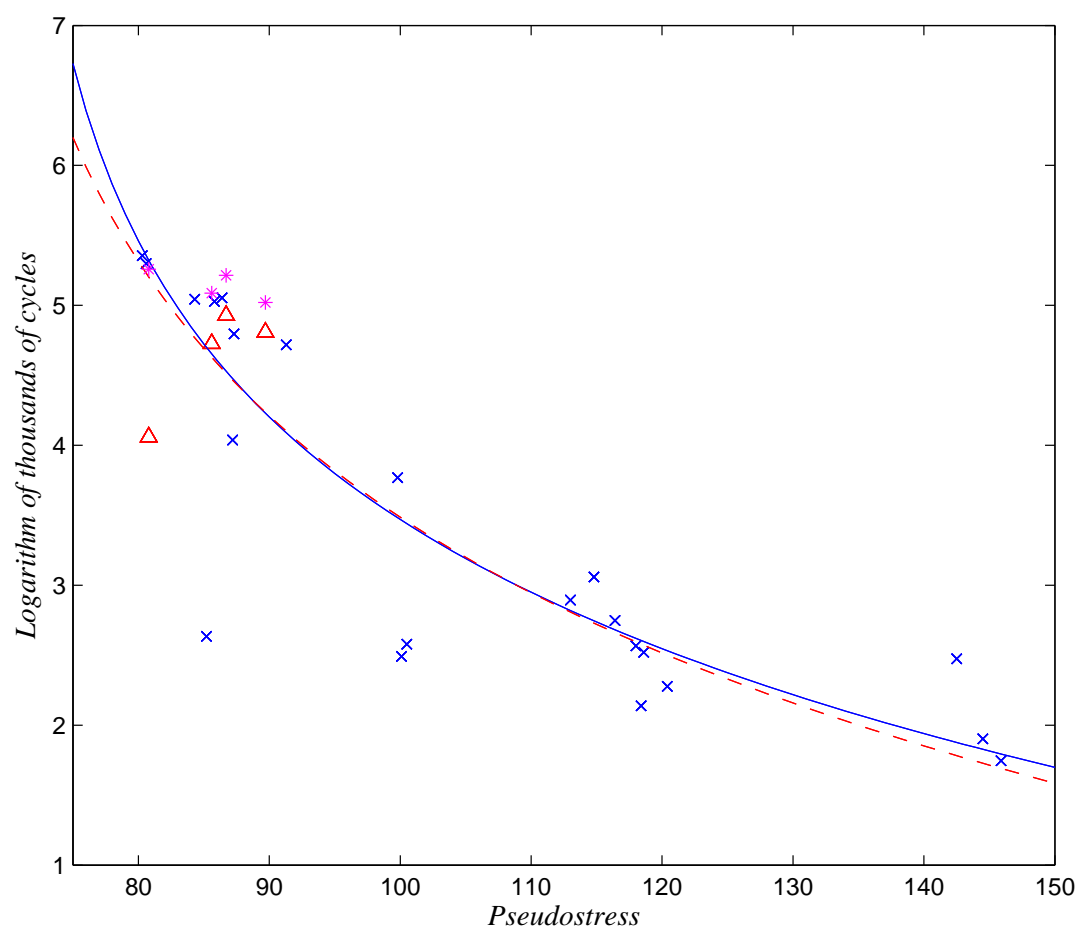

Figure 3: Nonlinear regression for the fatigue data. The solid, respectively, dashed line represents the estimated regression curve for the new method, respectively, Stute's method. Uncensored data points are given by $\times$, and censored observations by $\triangle$. The new data points obtained from the new method are represented by $*$. 


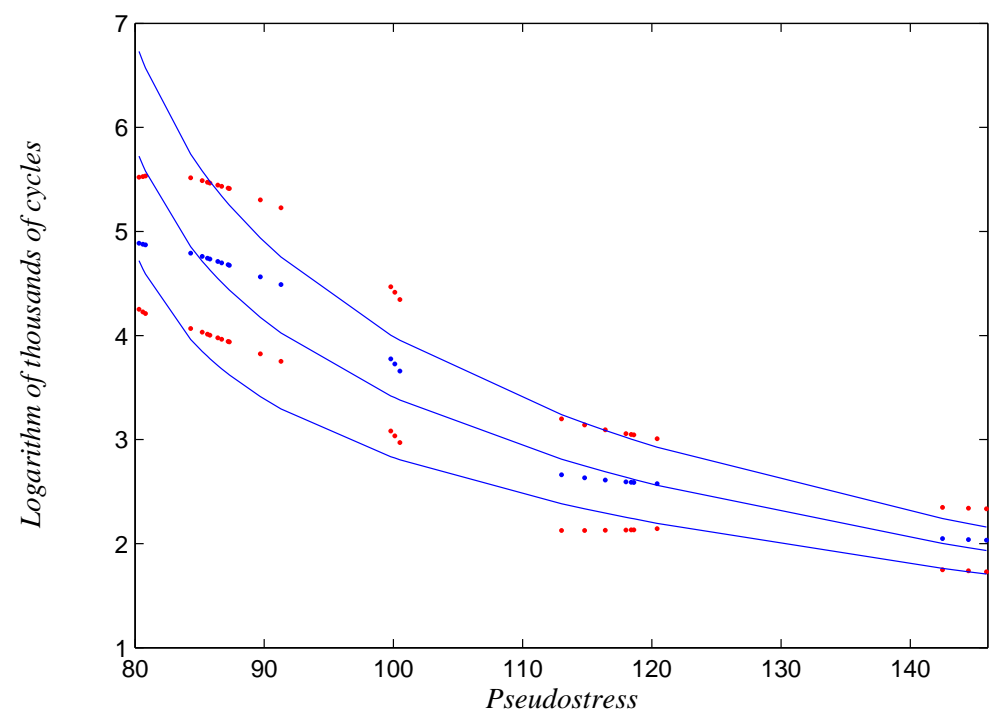

Figure 4: Parametric and nonparametric estimations of the conditional means and standard deviations for the fatigue data. Solid lines, respectively, dots, represent the parametric curves of Pascual and Meeker (1997) (where the conditional standard deviation is added and subtracted to the conditional mean), respectively, the corresponding pointwise nonparametric estimations explained above.

The parameter $\gamma$ in model (5.1) can be interpreted as a fatigue limit parameter i.e. specimens tested below this fatigue limit level of stress will never fail. Therefore, this parameter is constrained to be positive. The data set is then analyzed by means of Stute's procedure and the new method. For the computation of the variance, an asymptotic formula is used for Stute's method while for the new method we make use of a bootstrap approximation (see Subsection 4.1 for more details). Finally, confidence intervals are constructed for each parameter.

The results obtained by Stute's method are $\hat{\beta}_{0 s}=11.0474, \hat{\beta}_{1 s}=-2.1315, \hat{\gamma}_{s}=$ 65.2730 with estimated variances $13.6611 ; 0.6587 ; 120.5972$ respectively, while the new method provides $\hat{\beta}_{0 n}=9.2432, \hat{\beta}_{1 n}=-1.7221, \hat{\gamma}_{n}=71.1797$ with estimated variances $9.3389 ; 0.4100 ; 116.4890$ respectively. Confidence intervals for Stute's method are $\beta_{0} \in[3.8031,18.2917], \beta_{1} \in[-3.7222,-0.5408]$, and $\gamma \in[43.7489,86.7971]$. For the new method, confidence intervals based on the normal approximation are $\beta_{0} \in$ [3.6009; 15.5802], $\beta_{1} \in[-3.0564 ;-0.5463]$ and $\gamma \in[48.9448 ; 91.2534]$, and intervals obtained with the bootstrap percentile method are $\beta_{0} \in[6.7985 ; 19.0904], \beta_{1} \in[-3.7492$; $-1.1587]$ and $\gamma \in[33.4215 ; 77.9497]$. Graphs of the estimated curves are given in Figure 3. Nonparametric methods are also illustrated in Figure 4 together with the results 
of Pascual and Meeker (1997). The bandwidth is chosen by a bootstrap procedure (see Heuchenne and Van Keilegom (2005b) for details). A measure of the distance between those curves (often using a smoothed version of the parametric curves) could be established to validate Pascual and Meeker's (1997) forms.

\section{Conclusion}

In this paper, we introduce a new method to estimate the parameters of nonlinear censored regression models (1.1) where no parametric form is assumed for $\sigma^{2}(X)$ and the distribution of $\varepsilon$. This is interesting in applications like fatigue life analysis since existing models in this area impose parametric assumptions on these two quantitites. Compared to Stute's method (the other method with no parameter in the error term), it seems that the practical new choice of a bandwidth parameter combined with restrictive assumptions of Stute's estimator enable to obtain better results. Moreover, the conditional variance of the response can be estimated nonparametrically and used to obtain a parametric form. An important further work, in this context (here displayed in fatigue life analysis), should be to construct goodness-of-fit tests to validate parametric forms of $\sigma^{2}(\cdot)$. However, contrarily to Stute's procedure, the new one is not theoretically extended to the multiple regression case.

The method proposed in this paper can be extended to other loss functions by replacing the sum of squares (2.6) by a sum of absolute values or a combination of both (Huber loss functions). So, the method could be adapted to robust regression and more generally to M-estimation. Also, parametric estimation of conditional quantiles or truncated means could be considered instead of conditional means. This could be achieved by replacing new data points in (2.6) by L-functional estimators proposed by Heuchenne and Van Keilegom (2005a) and (2005b). Finally, it is also reasonable to think that some extensions to particular multiple regression problems can be quickly obtained (single index models, partial parametric models...).

\section{Appendix : Proofs of main results}

The following notations are needed in the statement of the asymptotic results given in Section 3.

$$
\xi_{\varepsilon}(z, \delta, y)=\left(1-F_{\varepsilon}^{0}(y)\right)\left\{-\int_{-\infty}^{y \wedge z} \frac{d H_{\varepsilon 1}^{0}(s)}{\left(1-H_{\varepsilon}^{0}(s)\right)^{2}}+\frac{I(z \leq y, \delta=1)}{1-H_{\varepsilon}^{0}(z)}\right\},
$$




$$
\begin{aligned}
& \xi(z, \delta, y \mid x)=(1-F(y \mid x))\left\{-\int_{-\infty}^{y \wedge z} \frac{d H_{1}(s \mid x)}{(1-H(s \mid x))^{2}}+\frac{I(z \leq y, \delta=1)}{1-H(z \mid x)}\right\} \\
& \eta(z, \delta \mid x)=\int_{-\infty}^{+\infty} \xi(z, \delta, v \mid x) J(F(v \mid x)) d v \sigma^{0}(x)^{-1}, \\
& \zeta(z, \delta \mid x)=\int_{-\infty}^{+\infty} \xi(z, \delta, v \mid x) J(F(v \mid x)) \frac{v-m^{0}(x)}{\sigma^{0}(x)} d v \sigma^{0}(x)^{-1} \\
& \gamma_{1}(y \mid x)=\int_{-\infty}^{y} \frac{h_{\varepsilon}^{0}(s \mid x)}{\left(1-H_{\varepsilon}^{0}(s)\right)^{2}} d H_{\varepsilon 1}^{0}(s)+\int_{-\infty}^{y} \frac{d h_{\varepsilon 1}^{0}(s \mid x)}{1-H_{\varepsilon}^{0}(s)} \\
& \gamma_{2}(y \mid x)=\int_{-\infty}^{y} \frac{s h_{\varepsilon}^{0}(s \mid x)}{\left(1-H_{\varepsilon}^{0}(s)\right)^{2}} d H_{\varepsilon 1}^{0}(s)+\int_{-\infty}^{y} \frac{d\left(s h_{\varepsilon 1}^{0}(s \mid x)\right)}{1-H_{\varepsilon}^{0}(s)} \\
& \varphi(x, z, \delta, y)=\xi_{\varepsilon}\left(\frac{z-m^{0}(x)}{\sigma^{0}(x)}, \delta, y\right)-S_{\varepsilon}^{0}(y) \eta(z, \delta \mid x) \gamma_{1}(y \mid x)-S_{\varepsilon}^{0}(y) \zeta(z, \delta \mid x) \gamma_{2}(y \mid x), \\
& \alpha_{i}(v)=\frac{\int_{v}^{S_{i}} u d F_{\varepsilon}^{0}(u)}{1-F_{\varepsilon}^{0}(v)}, \\
& B_{k}\left(z, Z_{j}, \Delta_{j} \mid X_{i}\right) \\
& =\frac{\partial m_{\theta_{0}^{T}}\left(X_{i}\right)}{\partial \theta_{k}} f_{X}^{-1}\left(X_{i}\right) \sigma^{0}\left(X_{i}\right)\left\{\left[\alpha_{i}^{\prime}\left(e_{i}^{0 T}(z)\right)-1+\frac{S_{i} f_{\varepsilon}^{0}\left(S_{i}\right)}{1-F_{\varepsilon}^{0}\left(e_{i}^{0 T}(z)\right)}\right] \eta\left(Z_{j}, \Delta_{j} \mid X_{i}\right)\right. \\
& \left.+\left[e_{i}^{0 T}(z) \alpha_{i}^{\prime}\left(e_{i}^{0 T}(z)\right)-\alpha_{i}\left(e_{i}^{0 T}(z)\right)+\frac{S_{i}^{2} f_{\varepsilon}^{0}\left(S_{i}\right)}{1-F_{\varepsilon}^{0}\left(e_{i}^{0 T}(z)\right)}\right] \zeta\left(Z_{j}, \Delta_{j} \mid X_{i}\right)\right\},
\end{aligned}
$$

$k=1, \ldots, d, i, j=1, \ldots, n$, where $S_{i}=S_{X_{i}}, e_{i}^{0 T}(z)=e_{X_{i}}^{0 T}(z)$ and for any $x \in R_{X}$, $S_{x}=\left(T_{x}-m^{0}(x)\right) / \sigma^{0}(x)$ and $e_{x}^{0 T}(z)=\left(z \wedge T_{x}-m^{0}(x)\right) / \sigma^{0}(x)$.

Let $\tilde{T}_{x}$ be any value less than the upper bound of the support of $H(\cdot \mid x)$ such that $\inf _{x \in R_{X}}\left(1-H\left(\tilde{T}_{x} \mid x\right)\right)>0$. For a (sub)distribution function $L(y \mid x)$ we will use the notations $l(y \mid x)=L^{\prime}(y \mid x)=(\partial / \partial y) L(y \mid x), \dot{L}(y \mid x)=(\partial / \partial x) L(y \mid x)$ and similar notations will be used for higher order derivatives.

The assumptions needed for the results of Section 3 are listed below.

$(A 1)(i) n a_{n}^{4} \rightarrow 0$ and $n a_{n}^{3+2 \delta}\left(\log a_{n}^{-1}\right)^{-1} \rightarrow \infty$ for some $\delta<1 / 2$.

(ii) $R_{X}$ is a compact interval.

(iii) $K$ is a density with compact support, $\int u K(u) d u=0$ and $K$ is twice continuously differentiable.

(iv) $\Omega$ is non-singular.

$(A 2)(i)$ There exist $0 \leq s_{0} \leq s_{1} \leq 1$ such that $s_{1} \leq \inf _{x} F\left(\tilde{T}_{x} \mid x\right), s_{0} \leq \inf \{s \in$ 
$[0,1] ; J(s) \neq 0\}, s_{1} \geq \sup \{s \in[0,1] ; J(s) \neq 0\}$ and $\inf _{x \in R_{X}} \inf _{s_{0} \leq s \leq s_{1}} f\left(F^{-1}(s \mid x) \mid x\right)>0$.

(ii) $J$ is twice continuously differentiable, $\int_{0}^{1} J(s) d s=1$ and $J(s) \geq 0$ for all $0 \leq s \leq 1$.

(iii) The function $x \rightarrow T_{x}\left(x \in R_{X}\right)$ is twice continuously differentiable.

$(A 3)(i) F_{X}$ is three times continuously differentiable and $\inf _{x \in R_{X}} f_{X}(x)>0$.

(ii) $m^{0}$ and $\sigma^{0}$ are twice continuously differentiable and $\inf _{x \in R_{X}} \sigma^{0}(x)>0$.

(iii) $E\left[\varepsilon^{02}\right]<\infty$ and $E\left[E^{04}\right]<\infty$.

$(A 4)(i) \eta(z, \delta \mid x)$ and $\zeta(z, \delta \mid x)$ are twice continuously differentiable with respect to $x$ and their first and second derivatives (with respect to $x$ ) are bounded, uniformly in $x \in R_{X}$, $z<\tilde{T}_{x}$ and $\delta$.

(ii) The first derivatives of $\eta(z, \delta \mid x)$ and $\zeta(z, \delta \mid x)$ with respect to $z$ are of bounded variation and the variation norms are uniformly bounded over all $x$.

(A5) The function $y \rightarrow P\left(m^{0}(X)+e \sigma^{0}(X) \leq y\right)(y \in \mathbb{R})$ is differentiable for all $e \in \mathbb{R}$ and the derivative is uniformly bounded over all $e \in \mathbb{R}$.

(A6) For $L(y \mid x)=H(y \mid x), H_{1}(y \mid x), H_{\varepsilon}^{0}(y \mid x)$ or $H_{\varepsilon 1}^{0}(y \mid x): L^{\prime}(y \mid x)$ is continuous in $(x, y)$ and $\sup _{x, y}\left|y^{2} L^{\prime}(y \mid x)\right|<\infty$, the same holds for all other partial derivatives of $L(y \mid x)$ with respect to $x$ and $y$ up to order three, and $\sup _{x, y}\left|y^{3} L^{\prime \prime \prime}(y \mid x)\right|<\infty$.

$(A 7)(i) \sup _{x, z} \int\left|B_{k}^{\prime}(t, z, \delta \mid x)\right| h(t) d t<\infty(k=1, \ldots, d ; \delta=0,1)$.

(ii) $\sup _{z} \int \sup _{x}\left|B_{k}^{\prime \prime}(t, z, \delta \mid x)\right| h(t) d t<\infty(k=1, \ldots, d ; \delta=0,1)$, where $B_{k}^{\prime\left({ }^{\prime}\right)}(t, z, \delta \mid x)$ equals the first (second) derivative of $B_{k}(t, z, \delta \mid x)$ with respect to $x$ when $t \neq T_{x}$ and equals 0 otherwise.

(A8) For the density $f_{X \mid Z, \Delta}(x \mid z, \delta)$ of $X$ given $(Z, \Delta), \sup _{x, z}\left|f_{X \mid Z, \Delta}(x \mid z, \delta)\right|<\infty$, $\sup _{x, z}\left|\dot{f}_{X \mid Z, \Delta}(x \mid z, \delta)\right|<\infty, \sup _{x, z}\left|\ddot{f}_{X \mid Z, \Delta}(x \mid z, \delta)\right|<\infty(\delta=0,1)$.

$(A 9) \Theta$ is compact and $\theta_{0}^{T}$ is an interior point of $\Theta$. All partial derivatives of $m_{\theta}(x)$ with respect to the components of $\theta$ up to order three exist and are continuous in $(x, \theta)$ for all $x$ and $\theta$.

(A10) The function $E\left[\left\{E\left(Y_{T}^{*} \mid X\right)-m_{\theta}(X)\right\}^{2}\right]$ has a unique minimum in $\theta=\theta_{0}^{T}$.

Proof of Theorem 3.1. We prove the consistency of $\hat{\theta}_{n}^{T}$ by verifying the conditions of Theorem 5.7 in van der Vaart (1998, p. 45). From the definition of $\hat{\theta}_{n}^{T}$ and condition $(A 10)$, it follows that it suffices to show that

$$
\sup _{\theta}\left|S_{n}(\theta)-S_{0}(\theta)\right| \rightarrow_{P} 0,
$$

where $S_{0}(\theta)=E\left[\sigma^{02}(X) \operatorname{Var}\left(\varepsilon_{T}^{0 *} \mid X\right)\right]+E\left[\left\{E\left(Y_{T}^{*} \mid X\right)-m_{\theta}(X)\right\}^{2}\right]$. The second statement 
of Theorem 3.1 then follows immediately from (A.1) together with the consistency of $\hat{\theta}_{n}^{T}$. To prove (A.1) we write

$$
\begin{aligned}
S_{n}(\theta) & =\frac{1}{n} \sum_{i=1}^{n}\left(\hat{Y}_{T i}^{*}-Y_{T i}^{*}\right)^{2}+\frac{1}{n} \sum_{i=1}^{n}\left(Y_{T i}^{*}-m_{\theta}\left(X_{i}\right)\right)^{2}+\frac{2}{n} \sum_{i=1}^{n}\left(\hat{Y}_{T i}^{*}-Y_{T i}^{*}\right)\left(Y_{T i}^{*}-m_{\theta}\left(X_{i}\right)\right) \\
& =S_{n 1}+S_{n 2}(\theta)+S_{n 3}(\theta) .
\end{aligned}
$$

In order to treat $S_{n 1}$ and $S_{n 3}(\theta)$, we first consider the difference

$$
\begin{aligned}
\hat{Y}_{T i}^{*}-Y_{T i}^{*}= & \left\{\left[\hat{m}^{0}\left(X_{i}\right)-m^{0}\left(X_{i}\right)\right]+\frac{\hat{\sigma}^{0}\left(X_{i}\right)}{1-\hat{F}_{\varepsilon}^{0}\left(\hat{E}_{i}^{0 T}\right)} \int_{\hat{E}_{i}^{0 T}}^{\hat{S}_{i}} u d \hat{F}_{\varepsilon}^{0}(u)\right. \\
& \left.-\frac{\sigma^{0}\left(X_{i}\right)}{1-F_{\varepsilon}^{0}\left(E_{i}^{0 T}\right)} \int_{E_{i}^{0 T}}^{S_{i}} u d F_{\varepsilon}^{0}(u)\right\}\left(1-\Delta_{i}\right) \\
= & \left\{A_{1 i}+A_{2 i}+A_{3 i}\right\}\left(1-\Delta_{i}\right) .
\end{aligned}
$$

Using Proposition 4.5 of Van Keilegom and Akritas (1999) (hereafter abbreviated by VKA $), A_{1 i}=O\left(\left(n a_{n}\right)^{-1 / 2}\left(\log a_{n}^{-1}\right)^{1 / 2}\right)$ a.s. uniformly in $i$. Next, write

$$
\begin{aligned}
A_{2 i}+A_{3 i} \\
=\frac{\hat{\sigma}^{0}\left(X_{i}\right)-\sigma^{0}\left(X_{i}\right)}{1-\hat{F}_{\varepsilon}^{0}\left(\hat{E}_{i}^{0 T}\right)} \int_{\hat{E}_{i}^{0 T}}^{\hat{S}_{i}} u d \hat{F}_{\varepsilon}^{0}(u)+\sigma^{0}\left(X_{i}\right) \frac{\hat{F}_{\varepsilon}^{0}\left(\hat{E}_{i}^{0 T}\right)-F_{\varepsilon}^{0}\left(E_{i}^{0 T}\right)}{\left(1-\hat{F}_{\varepsilon}^{0}\left(\hat{E}_{i}^{0 T}\right)\right)\left(1-F_{\varepsilon}^{0}\left(E_{i}^{0 T}\right)\right)} \int_{\hat{E}_{i}^{0 T}}^{\hat{S}_{i}} u d \hat{F}_{\varepsilon}^{0}(u) \\
\quad+\frac{\sigma^{0}\left(X_{i}\right)}{1-F_{\varepsilon}^{0}\left(E_{i}^{0 T}\right)} \int_{\hat{E}_{i}^{0 T}}^{E_{i}^{0 T}} u d \hat{F}_{\varepsilon}^{0}(u)+\frac{\sigma^{0}\left(X_{i}\right)}{1-F_{\varepsilon}^{0}\left(E_{i}^{0 T}\right)} \int_{E_{i}^{0 T}}^{S_{i}} u d\left(\hat{F}_{\varepsilon}^{0}(u)-F_{\varepsilon}^{0}(u)\right) \\
\quad+\frac{\sigma^{0}\left(X_{i}\right)}{1-F_{\varepsilon}^{0}\left(E_{i}^{0 T}\right)} \int_{S_{i}}^{\hat{S}_{i}} u d \hat{F}_{\varepsilon}^{0}(u) \\
=\sum_{j=1}^{5} B_{j i} .
\end{aligned}
$$

We will now prove the convergence to zero of each of these five terms. First, by using integration by parts, we can write

$$
\begin{aligned}
\int_{\hat{E}_{i}^{0 T}}^{E_{i}^{0 T}} u d \hat{F}_{\varepsilon}^{0}(u)= & E_{i}^{0 T}\left[\hat{F}_{\varepsilon}^{0}\left(E_{i}^{0 T}\right)-F_{\varepsilon}^{0}\left(E_{i}^{0 T}\right)\right]+\left[E_{i}^{0 T} F_{\varepsilon}^{0}\left(E_{i}^{0 T}\right)-\hat{E}_{i}^{0 T} F_{\varepsilon}^{0}\left(E_{i}^{0 T}\right)\right] \\
& +\hat{E}_{i}^{0 T}\left[F_{\varepsilon}^{0}\left(E_{i}^{0 T}\right)-\hat{F}_{\varepsilon}^{0}\left(\hat{E}_{i}^{0 T}\right)\right]-\int_{\hat{E}_{i}^{0 T}}^{E_{i}^{0 T}} \hat{F}_{\varepsilon}^{0}(u) d u
\end{aligned}
$$

By Corollary 3.2 in VKA (1999), the first term of (A.2) is $\left|E_{i}^{0 T}\right| O_{P}\left(n^{-1 / 2}\right)$, while from Proposition 4.5 in VKA (1999), it follows that the second and fourth term are $\left|E_{i}^{0 T}\right| O\left(\left(n a_{n}\right)^{-1 / 2}\left(\log a_{n}^{-1}\right)^{1 / 2}\right)$ a.s. The third term is $\left|E_{i}^{0 T}\right| O_{P}\left(\left(n a_{n}\right)^{-1 / 2}\left(\log a_{n}^{-1}\right)^{1 / 2}\right)$ using Proposition 4.5 of VKA (1999) and the fact that

$$
\sup _{x, z}\left|\hat{F}_{\varepsilon}^{0}\left\{\frac{z \wedge T_{x}-\hat{m}^{0}(x)}{\hat{\sigma}^{0}(x)}\right\}-F_{\varepsilon}^{0}\left\{\frac{z \wedge T_{x}-m^{0}(x)}{\sigma^{0}(x)}\right\}\right|=O_{P}\left(\left(n a_{n}\right)^{-1 / 2}\left(\log a_{n}^{-1}\right)^{1 / 2}\right) .
$$


This can be obtained as follows.

$$
\begin{aligned}
\hat{F}_{\varepsilon}^{0}\left\{\frac{z \wedge T_{x}-\hat{m}^{0}(x)}{\hat{\sigma}^{0}(x)}\right\}-F_{\varepsilon}^{0}\left\{\frac{z \wedge T_{x}-m^{0}(x)}{\sigma^{0}(x)}\right\} \\
=\hat{F}_{\varepsilon}^{0}\left\{\frac{z \wedge T_{x}-\hat{m}^{0}(x)}{\hat{\sigma}^{0}(x)}\right\}-F_{\varepsilon}^{0}\left\{\frac{z \wedge T_{x}-\hat{m}^{0}(x)}{\hat{\sigma}^{0}(x)}\right\} \\
\quad+F_{\varepsilon}^{0}\left\{\frac{z \wedge T_{x}-\hat{m}^{0}(x)}{\hat{\sigma}^{0}(x)}\right\}-F_{\varepsilon}^{0}\left\{\frac{z \wedge T_{x}-m^{0}(x)}{\sigma^{0}(x)}\right\} \\
=\alpha_{n}^{1}(z, x)+\alpha_{n}^{2}(z, x) .
\end{aligned}
$$

Using Corollary 3.2 of VKA (1999), $\sup _{x, z}\left|\alpha_{n}^{1}(z, x)\right|$ is $O_{P}\left(n^{-1 / 2}\right)$. For $\alpha_{n}^{2}(z, x)$, we use two first order Taylor developments :

$$
\alpha_{n}^{2}(z, x)=-\frac{\hat{m}^{0}(x)-m^{0}(x)}{\hat{\sigma}^{0}(x)} f_{\varepsilon}^{0}\left(A_{x}\right)-\frac{\hat{\sigma}^{0}(x)-\sigma^{0}(x)}{\hat{\sigma}^{0}(x)} \frac{z \wedge T_{x}-m^{0}(x)}{\sigma^{0}(x)} f_{\varepsilon}^{0}\left(B_{x}\right),
$$

for some $A_{x}\left(B_{x}\right)$ between $\frac{z \wedge T_{x}-m^{0}(x)}{\hat{\sigma}^{0}(x)}$ and $\frac{z \wedge T_{x}-\hat{m}^{0}(x)}{\hat{\sigma}^{0}(x)}\left(\frac{z \wedge T_{x}-m^{0}(x)}{\sigma^{0}(x)}\right.$ and $\left.\frac{z \wedge T_{x}-m^{0}(x)}{\hat{\sigma}^{0}(x)}\right)$. Using Proposition 4.5 of VKA (1999) and the fact that $\sup _{e}\left|e f_{\varepsilon}^{0}(e)\right|<+\infty, \alpha_{n}^{2}(z, x)=$ $O\left(\left(n a_{n}\right)^{-1 / 2}\left(\log a_{n}^{-1}\right)^{1 / 2}\right)$ a.s. Hence, $B_{3 i}=\left|E_{i}^{0 T}\right| O_{P}\left(\left(n a_{n}\right)^{-1 / 2}\left(\log a_{n}^{-1}\right)^{1 / 2}\right)$. In a similar way it can be shown that $B_{5 i}=O_{P}\left(\left(n a_{n}\right)^{-1 / 2}\left(\log a_{n}^{-1}\right)^{1 / 2}\right)$. Using Lemma A.1 of Heuchenne and Van Keilegom (2004), it follows that $\int_{E_{i}^{0 T}}^{S_{i}} u d \hat{F}_{\varepsilon}^{0}(u)=O_{P}(1)$. Hence, combining this with the rates obtained for $B_{3 i}$ and $B_{5 i}$ we get that $\int_{\hat{E}_{i}^{0 T}}^{\hat{S}_{i}} u d \hat{F}_{\varepsilon}^{0}(u)=\left|E_{i}^{0 T}\right| O_{P}(1)$. Therefore, using Proposition 4.5 in VKA (1999) and the uniform consistency of $\hat{F}_{\varepsilon}^{0}\left(\hat{E}_{i}^{0 T}\right)$, $B_{1 i}$ and $B_{2 i}$ are $\left|E_{i}^{0 T}\right| O_{P}\left(\left(n a_{n}\right)^{-1 / 2}\left(\log a_{n}^{-1}\right)^{1 / 2}\right)$. Finally,

$$
\begin{aligned}
B_{4 i}= & \frac{\sigma^{0}\left(X_{i}\right)}{1-F_{\varepsilon}^{0}\left(E_{i}^{0 T}\right)}\left\{S_{i}\left[\hat{F}_{\varepsilon}^{0}\left(S_{i}\right)-F_{\varepsilon}^{0}\left(S_{i}\right)\right]-E_{i}^{0 T}\left[\hat{F}_{\varepsilon}^{0}\left(E_{i}^{0 T}\right)-F_{\varepsilon}^{0}\left(E_{i}^{0 T}\right)\right]\right. \\
& \left.-\int_{E_{i}^{0 T}}^{S_{i}}\left(\hat{F}_{\varepsilon}^{0}(u)-F_{\varepsilon}^{0}(u)\right) d u\right\} \\
= & \left|E_{i}^{0 T}\right| O_{P}\left(n^{-1 / 2}\right),
\end{aligned}
$$

such that

$$
\hat{Y}_{T i}^{*}-Y_{T i}^{*}=\left|E_{i}^{0 T}\right| O_{P}\left(\left(n a_{n}\right)^{-1 / 2}\left(\log a_{n}^{-1}\right)^{1 / 2}\right) .
$$

Therefore, $\left|S_{n 1}\right|=O_{P}\left(\left(n a_{n}\right)^{-1} \log a_{n}^{-1}\right)$. In the same way, using (A.3) and the continuity of $m_{\theta}(x)$ on $R_{X} \times \Theta, \sup _{\theta}\left|S_{n 3}(\theta)\right|=O_{P}\left(\left(n a_{n}\right)^{-1 / 2}\left(\log a_{n}^{-1}\right)^{1 / 2}\right)$. For $S_{n 2}(\theta)$, write

$$
\begin{aligned}
S_{n 2}(\theta)= & \frac{1}{n} \sum_{i=1}^{n}\left\{Y_{T i}^{*}-E\left(Y_{T i}^{*} \mid X_{i}\right)\right\}^{2}+\frac{1}{n} \sum_{i=1}^{n}\left\{E\left(Y_{T i}^{*} \mid X_{i}\right)-m_{\theta}\left(X_{i}\right)\right\}^{2} \\
& +\frac{2}{n} \sum_{i=1}^{n}\left\{Y_{T i}^{*}-E\left(Y_{T i}^{*} \mid X_{i}\right)\right\}\left\{E\left(Y_{T i}^{*} \mid X_{i}\right)-m_{\theta}\left(X_{i}\right)\right\}=S_{n 21}+S_{n 22}(\theta)+S_{n 23}(\theta) .
\end{aligned}
$$


Since $E\left[\varepsilon^{02}\right]<\infty$, it is easily seen that

$$
S_{n 21}=E\left[\sigma^{02}(X)\left\{\varepsilon_{0 T}^{*}-E\left(\varepsilon_{0 T}^{*} \mid X\right)\right\}^{2}\right]+o(1) \text { a.s. }
$$

For $S_{n 22}(\theta)$ and $S_{n 23}(\theta)$ we use Theorem 2 of Jennrich (1969). The function $g$ in this theorem is given by $g_{\theta}(x)=\left\{E\left(Y_{T}^{*} \mid x\right)-m_{\theta}(x)\right\}^{2}$ for $S_{n 22}(\theta)$ and

$$
\begin{aligned}
g_{\theta}(z, \delta, x)= & {\left[z \delta+(1-\delta)\left\{m^{0}(x)+\frac{\sigma^{0}(x)}{1-F_{\varepsilon}^{0}\left(\frac{z \wedge T_{x}-m^{0}(x)}{\sigma^{0}(x)}\right)} \int_{\frac{z \wedge T_{x}-m^{0}(x)}{\sigma^{0}(x)}}^{\frac{T_{x}-m^{0}(x)}{\sigma^{0}(x)}} u d F_{\varepsilon}^{0}(u)\right\}-E\left(Y_{T}^{*} \mid x\right)\right] } \\
& \times\left\{E\left(Y_{T}^{*} \mid x\right)-m_{\theta}(x)\right\}
\end{aligned}
$$

for $S_{n 23}(\theta)$. Since $E\left|\varepsilon^{0}\right|<\infty,\left|g_{\theta}(x)\right| \leq C_{1}$ and $\left|g_{\theta}(z, \delta, x)\right| \leq h(z, \delta)=C_{2} z \delta+C_{3}$ for some $C_{1}, C_{2}, C_{3}>0$, and for all $(z, \delta, x)$ and $\theta$, where $h$ is integrable with respect to the joint distribution of $(z, \delta, x)$. From this,

$$
\sup _{\theta \in \Theta}\left|S_{n}(\theta)-E\left[\sigma^{02}(X) \operatorname{Var}\left(\varepsilon_{0 T}^{*} \mid X\right)\right]-E\left[\left\{E\left(Y_{T}^{*} \mid X\right)-m_{\theta}(X)\right\}^{2}\right]\right|=o_{P}(1) .
$$

This finishes the proof.

Proof of Theorem 3.2. For some $\theta_{1 n}$ between $\hat{\theta}_{n}^{T}$ and $\theta_{0}^{T}$

$$
\hat{\theta}_{n}^{T}-\theta_{0}^{T}=-\left\{\frac{\partial^{2} S_{n}\left(\theta_{1 n}\right)}{\partial \theta \partial \theta^{\prime}}\right\}^{-1} \frac{\partial S_{n}\left(\theta_{0}^{T}\right)}{\partial \theta}=-R_{1}^{-1} R_{2} .
$$

We have

$$
R_{2}=-\frac{2}{n} \sum_{i=1}^{n}\left(\hat{Y}_{T_{i}}^{*}-Y_{T_{i}}^{*}\right) \frac{\partial m_{\theta_{0}^{T}}\left(X_{i}\right)}{\partial \theta}-\frac{2}{n} \sum_{i=1}^{n}\left\{Y_{T_{i}}^{*}-m_{\theta_{0}^{T}}\left(X_{i}\right)\right\} \frac{\partial m_{\theta_{0}^{T}}\left(X_{i}\right)}{\partial \theta}=R_{21}+R_{22},
$$

such that $R_{22}$ is a sum of i.i.d. random variables with zero mean (by definition of $\theta_{0}^{T}$ ). For each component $j$ of $R_{21}$, we use a technique similar to Theorem 1 of Heuchenne and Van Keilegom (2004) to obtain an asymptotic representation. So we obtain

$$
\begin{aligned}
R_{21 j}=- & \frac{2}{n} \sum_{i=1}^{n} \int_{R_{X}} \frac{\partial m_{\theta_{0}^{T}}(x)}{\partial \theta_{j}} \sigma(x) \int_{-\infty}^{+\infty}\left\{\frac{\varphi\left(X_{i}, Z_{i}, \Delta_{i}, e_{x}^{0 T}(z)\right)}{\left\{1-F_{\varepsilon}^{0}\left(e_{x}^{0 T}(z)\right)\right\}^{2}} \int_{e_{x}^{0 T}(z)}^{S_{x}} u d F_{\varepsilon}^{0}(u)\right. \\
& \left.+\frac{1}{1-F_{\varepsilon}^{0}\left(e_{x}^{0 T}(z)\right)} \int_{e_{x}^{0 T}(z)}^{S_{x}} u d \varphi\left(X_{i}, Z_{i}, \Delta_{i}, u\right)\right\} d H_{0}(z \mid x) d F_{X}(x) \\
& +f_{X}\left(X_{i}\right) \int_{-\infty}^{\infty} B_{j}\left(z, Z_{i}, \Delta_{i} \mid X_{i}\right) d H_{0}\left(z \mid X_{i}\right)+o_{P}\left(n^{-1 / 2}\right)
\end{aligned}
$$

$(j=1, \ldots, d ; i=1, \ldots, n)$. For $R_{1}$, we write

$$
\begin{aligned}
R_{1}=- & \frac{2}{n}\left\{\sum_{i=1}^{n}\left(\hat{Y}_{T_{i}}^{*}-Y_{T_{i}}^{*}\right) \frac{\partial^{2} m_{\theta_{1 n}}\left(X_{i}\right)}{\partial \theta \partial \theta^{\prime}}+\sum_{i=1}^{n}\left(Y_{T_{i}}^{*}-m_{\theta_{1 n}}\left(X_{i}\right)\right) \frac{\partial^{2} m_{\theta_{1 n}}\left(X_{i}\right)}{\partial \theta \partial \theta^{\prime}}\right. \\
& \left.-\sum_{i=1}^{n}\left(\frac{\partial m_{\theta_{1 n}}\left(X_{i}\right)}{\partial \theta}\right)\left(\frac{\partial m_{\theta_{1 n}}\left(X_{i}\right)}{\partial \theta^{\prime}}\right)\right\}=R_{11}+R_{12}+R_{13} .
\end{aligned}
$$


Using assumption (A9), the fact that $\left|\hat{Y}_{T_{i}}^{*}-Y_{T_{i}}^{*}\right|=\left|E_{i}^{0 T}\right| O_{P}\left(\left(n a_{n}\right)^{-1 / 2}\left(\log a_{n}^{-1}\right)^{1 / 2}\right)$ (see the proof of Theorem 3.1), we have that $R_{11}=o_{P}(1)$. Again using condition (A9),

$$
\begin{aligned}
R_{1} & =\frac{2}{n} \sum_{i=1}^{n} \frac{\partial m_{\theta_{0}^{T}}\left(X_{i}\right)}{\partial \theta}\left(\frac{\partial m_{\theta_{0}^{T}}\left(X_{i}\right)}{\partial \theta}\right)^{\prime}-\frac{2}{n} \sum_{i=1}^{n}\left\{Y_{T_{i}}^{*}-m_{\theta_{0}^{T}}\left(X_{i}\right)\right\} \frac{\partial^{2} m_{\theta_{0}^{T}}\left(X_{i}\right)}{\partial \theta \partial \theta^{\prime}}+o_{P}(1) \\
& =2 E\left[\frac{\partial m_{\theta_{0}^{T}}(X)}{\partial \theta}\left(\frac{\partial m_{\theta_{0}^{T}}(X)}{\partial \theta}\right)^{\prime}-\left\{Y_{T}^{*}-m_{\theta_{0}^{T}}(X)\right\} \frac{\partial^{2} m_{\theta_{0}^{T}}(X)}{\partial \theta \partial \theta^{\prime}}\right]+o_{P}(1) \\
& =2 \Omega+o_{P}(1) .
\end{aligned}
$$

The result now follows. 


\section{References}

Beran, R. (1981). Nonparametric regression with randomly censored survival data. Technical Report, Univ. California, Berkeley.

Efron, B. (1981). Censored data and the bootstrap. J. Amer. Statist. Assoc., 76, 312-319.

Heuchenne, C. (2005). Strong uniform consistency results of the weighted average of conditional artificial data points. (submitted paper)

Heuchenne, C. and Van Keilegom, I. (2004). Polynomial regression with censored data based on preliminary nonparametric estimation (conditionally accepted by Ann. Inst. Statist. Math.).

Heuchenne, C. and Van Keilegom, I. (2005a). Estimation in nonparametric location-scale regression models with censored data (submitted paper).

Heuchenne, C. and Van Keilegom, I. (2005b). Mean preservation in nonparametric regression with censored data (submitted paper).

Jennrich, R.I. (1969). Asymptotic properties of nonlinear least squares estimators. Ann. Math. Statist., 40, 633-643.

Kaplan, E. L. and Meier, P. (1958). Nonparametric estimation from incomplete observations. J. Amer. Statist. Assoc., 53, 457-481.

Levenberg, K. (1944). A method for the solution of certain problems in least squares. Quart. Appl. Math., 2, 164-168.

Li, G. and Datta, S. (2001). A bootstrap approach to non-parametric regression for right censored data. Ann. Inst. Statist. Math., 53, 708-729.

Marquardt, D. (1963). An algorithm for least-squares estimation of nonlinear parameters. SIAM J. Appl. Math., 11, 431-441.

Meeker, W.Q. and Escobar, L.A. (1998). Statistical methods for reliability data. Wiley, New-York.

Müller, H.-G. and Wang, J.-L. (1994). Hazard rate estimation under random censoring with varying kernels and bandwidths. Biometrics, 50, 61-76.

Nelson, W. (1984). Fitting of fatigue curves with nonconstant standard deviation to data with runouts. J. Testing Eval., 12, 69-77.

Pascual, F.G. and Meeker, W.Q. (1997). Analysis of fatigue data with runouts based on a model with nonconstant standard deviation and a fatigue limit parameter. J. Testing Eval., 25, 292-301.

Stute, W. (1993). Consistent estimation under random censorship when covariables are present. J. Multiv. Analysis, 45, 89-103.

Stute, W. (1999). Nonlinear censored regression. Statistica Sinica, 9, 1089-1102.

Van der Vaart, A.W. (1998). Asymptotic statistics. Cambridge University Press, Cambridge. 
Van Keilegom, I. and Akritas, M. G. (1999). Transfer of tail information in censored regression models. Ann. Statist., 27, 1745-1784.

Van Keilegom, I. and Veraverbeke, N. (1997). Estimation and bootstrap with censored data in fixed design nonparametric regression. Ann. Inst. Statist. Math., 49, 467-491. Address for correspondence :

Institut de Statistique

Université catholique de Louvain

Voie du Roman Pays, 20

B-1348 Louvain-la-Neuve

Belgium

Email: vankeilegom@stat.ucl.ac.be 